\title{
SOBREPESO Y OBESIDAD EN ADULTOS CON DIABETES MELLITUS TIPO 1
}

\section{OVERWEIGHT AND OBESITY IN ADULTS WITH TYPE 1 DIABETES MELLITUS}

\author{
Alejandra Cicchitti ${ }^{2,3}$, Celina Bertona ${ }^{2,3}$, Joaquín González ${ }^{1,2,3}$, Norma Carrasco ${ }^{2}$, Leticia Barrera ${ }^{4}$, \\ Raúl David ${ }^{5}$, Laura Romero ${ }^{6}$, Luis Biliato², Edgardo Trinajstic², Martín Rodríguez ${ }^{1,2}$. \\ En representación del Grupo de Estudio MENDODIAB-1
}

\section{RESUMEN}

Introducción: si bien la idea tradicional ha sido que los pacientes con diabetes mellitus tipo 1 (DM1) son delgados, la prevalencia de sobrepeso y obesidad ha aumentado en paralelo con la población general. El tratamiento intensivo disminuyó las complicaciones microvasculares pero generó sobreinsulinización, hipoglucemias, ingesta defensiva y ganancia de peso, con coexistencia de elementos del síndrome metabólico.

Objetivos: determinar en pacientes adultos con DM1 la frecuencia de sobrepeso, obesidad y los factores de riesgo cardiovascular y el grado de insulinorresistencia asociados.

Pacientes y métodos: se estratificaron 414 DM1 $\geq 18$ años, mujeres $52 \%$, edad $34,8 \pm 13,9$ años, de 14 departamentos de la provincia de Mendoza según IMC en normopeso, sobrepeso y obesidad. Se analizaron: edad, actividad física (AF) $\geq 3$ veces/semana, HbA1c, LDL-C $<100 \mathrm{mg} / \mathrm{dL}$, hipertensión arterial (HTA), nivel de instrucción y sensibilidad a la insulina según tasa estimada de disposición de glucosa (TeDG) por fórmula de Williams y Orchard.

Resultados: sobrepeso (S) presentó el 34,2\% y obesidad (O) el $10 \%$. Las mujeres mostraron mayor frecuencia de normopeso que los varones ( $62 \%$ vs $49 \% ; p=0,01)$, menor frecuencia de S $(28 \%$ vs $41 \% ; p=0,005)$ y sin diferencia de 0 ( $10 \%$ vs $9,5 \% ; p=0,87)$. Hubo aumento de $S / O$ con la edad $(p<0,0001)$. La presión arterial sistólica (PAS) $\geq 130 \mathrm{mmHg}$ fue más frecuente en $\mathrm{O}(54 \%)$, que en $\mathrm{S}(29 \%)$ y que en normopeso (16\%) $(p<0,0001)$. La TeDG fue de 9,14 $\pm 1,64 \mathrm{mg} / \mathrm{kg} / \mathrm{min}$ en normopeso, $7,62 \pm 1,67 \mathrm{mg} / \mathrm{kg} / \mathrm{min}$ en $\mathrm{S}$ y $6,02 \pm 2,20 \mathrm{mg} / \mathrm{kg} / \mathrm{min}$ en $\mathrm{O}(\mathrm{p}<0,0001)$. LDL-C $\leq 100 \mathrm{mg} / \mathrm{dL}$ fue porcentualmente más frecuente en normopeso (61\%) que en $S(51 \%)$ y que en $\mathrm{O}$ $(46 \%)$ sin alcanzar diferencia significativa $(p=0,08)$. No hubo diferencias significativas entre los tres grupos en $\mathrm{HbA1c}$, AF $\geq 3$ veces/semana ni en nivel de instrucción.

Conclusiones: la frecuencia de S/O en DM1 adultos (44,2\%) es alta y similar a observaciones recientes. El incremento es marcado entre los 25-50 años. El S/O se asociaron fuertemente con HTA e insulinorresistencia. El control del peso debe ser un objetivo prioritario en las personas con DM1.

Palabras clave: diabetes mellitus tipo 1; obesidad; sobrepeso.

\section{ABSTRACT}

Introduction: while the traditional idea has been that patients with type 1 diabetes mellitus (DM1) are thin, the prevalence of overweight and obesity has increased in parallel with the general population. Intensive treatment reduced microvascular complications, but generated overinsulinization, hypoglycemia, defensive intake and weight gain, with coexistence of elements of the metabolic syndrome.

Objectives: to determine the frequency of overweight, obesity and the associated risk factors and degree of insulin resistance in patients with DM1.

Patients and methods: 414 DM1 $\geq 18$ years, women 52\%, age $34.8 \pm 13.9$ years of 14 departments of the province of Mendoza were stratified according to $B M I$ in normal weight $N$, overweight and obesity. The following were analyzed: age, physical activity (PA) $\geq 3$ times/week, HbA1c, LDL-C <100 mg/ $d L, H B P$, level of education and insulin sensitivity according to the estimated rate of glucose disposition (ERGD) per Williams formula.

Results: overweight (OW) presented $34.2 \%$ and obesity (O) $10 \%$. Women presented a higher frequency of normal weight than men $(62 \%$ vs $49 \% ; p=0.01)$, lower frequency of OW $(28 \%$ vs $41 \% ; p=0.005)$ and without difference of $0(10 \%$ vs $9.5 \%$; $p=0.87)$. There was an increase in OW/O with age $(p<0.0001)$. The systolic blood pressure (SBP) $\geq 130 \mathrm{mmHg}$ was more frequent in $\mathrm{O}(54 \%)$ than in OW (29\%) and in normal weight (16\%) $(p<0.0001)$. The ERGD was $9.14 \pm 1.64 \mathrm{mg} / \mathrm{kg} / \mathrm{min}$ in the normal weight, $7.62 \pm 1.67 \mathrm{mg} / \mathrm{kg} / \mathrm{min}$ in the OW and $6.02 \pm 2.20 \mathrm{mg} / \mathrm{kg} / \mathrm{min}$ in the $O(p<0.0001)$. LDL-C $\leq 100 \mathrm{mg} / \mathrm{dL}$ was percentage more frequent in normal weight $(61 \%)$ than in OW $(51 \%)$ and in $O$ (46\%) without reaching significant difference $(p=0.08)$. There were no significant differences between the three groups in HbA1c, PA $\geq 3$ times/week or in the level of instruction.

Conclusions: the frequency of OW/O in adult DM1 (44.2\%) is high and like recent observations. The increase is marked between 25-50 years. OW/O was strongly associated with hypertension and insulin resistance. Weight control should be a priority goal in people with DM1.

Key words: type 1 diabetes mellitus; obesity; overweight.

Revista de la Sociedad Argentina de Diabetes 2019; Vol. 53 (109-116) 
Instituto de Clínica Médica y Diabetes, staff médico, Mendoza, Argentina

2 Docente, Facultad de Ciencias Médicas, Universidad Nacional de Cuyo, Mendoza, Argentina

3 Médico de Planta, Hospital Universitario, Universidad Nacional de Cuyo, Mendoza, Argentina

4 Obra Social de Empleados Públicos de Mendoza, staff médico, Mendoza, Argentina

5 Jefe de la Sección Diabetes Hospital Perrupato, San Martín, Mendoza, Argentina
6 Médico de Planta, Hospital Del Carmen, Godoy Cruz, Mendoza, Argentina

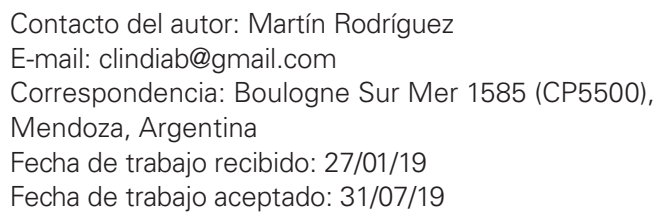

Conflictos de interés: los autores declaran que no existe conflicto de interés.

\section{INTRODUCCIÓN}

En los últimos 20 años la prevalencia de obesidad se ha triplicado en todo el mundo, por lo cual ya se considera una verdadera epidemia'. Si bien la idea tradicional ha sido que los pacientes con diabetes mellitus tipo 1 (DM1) son delgados, la prevalencia de sobrepeso y obesidad aumentó en paralelo con la población general. Incluso algunos autores refieren un ritmo de incremento más acelerado aún que el de la población general². El tratamiento intensificado disminuyó las complicaciones microvasculares pero generó sobreinsulinización, hipoglucemias, ingesta defensiva y ganancia de peso, con coexistencia de elementos del síndrome metabólico.

Es probable también que una mayor frecuencia de obesidad de base aumente la demanda de insulina y esto acelere la presentación de DM1 en sujetos genéticamente predispuestos (teoría del "acelerador" de Wilkin), lo que se ha dado en llamar "diabetes doble"3. Otros denominan "diabetes doble" a sujetos con DM1 con sobrepeso/obesidad que desarrollan estigmas de DM2 asociados a una marcada resistencia a la insulina exógena ${ }^{4}$. La expectativa de vida en personas con DM1 ha mejorado pero aún es $\approx 10$ años menor que la de la población general ${ }^{5}$. La enfermedad macrovascular es la principal causa de muerte en DM1 y es cinco veces más frecuente que en no DM16.

El problema se vislumbra ya desde la niñez y adolescencia. En siete estudios observacionales y de intervención en DM1 menores de 18 años, publicados entre 1990 y 2013, la prevalencia de sobrepeso/obesidad osciló entre el 12,5 y el 33,3\%?. El estudio SEARCH for Diabetes in Youth, utilizando medidas antropométricas realizadas bajo un protocolo estandarizado, encontró en Estados Unidos que el 22,1\% de los jóvenes con DM1 tenía sobrepeso en comparación con el 16,1\% de los jóvenes sin $\mathrm{DM}^{8}$. En un estudio que siguió a 625 pacientes adultos mayores de 18 años con DM1 y una edad media al comienzo de 29,1 años, durante un promedio de 18 años, la prevalencia de sobrepeso aumentó de 29 a $42 \%$ y la de obesidad siete veces de 3 a $23 \%{ }^{9}$. Más recientemente autores australianos detectaron una prevalencia de sobrepeso/obesidad en DM1 $\geq 18$ años $(n=501)$ del $38 \%$ de sobrepeso y $17 \%$ de obesidad ${ }^{10}$, y otro estudio sueco $(n=20.985)$ observó $35 \%$ de sobrepeso y 8,9\% de obesidad"11. En Córdoba (Argentina) Caeiro et al., en 446 DM1 115 años, hallaron $25 \%$ de sobrepeso y $15 \%$ de obesidad ${ }^{12}$.

El objetivo de este trabajo fue determinar la frecuencia de sobrepeso/obesidad en pacientes DM1 de la provincia de Mendoza. Dado el mayor riesgo cardiovascular de esta población se evaluó, además, la asociación del sobrepeso/obesidad con otros factores de riesgo cardiovascular: hipertensión arterial (HTA), LDL-C mayor de $100 \mathrm{mg} / \mathrm{dL}$, sedentarismo y grado de insulinorresistencia a través de la tasa estimada de disposición de glucosa (TeDG).

\section{PACIENTES Y MÉTODOS}

Por interrogatorio, examen físico y registro de historias clínicas, entre junio de 2017 y abril de 2018, se evaluaron a sujetos DM1 $\geq 18$ años, habitantes de 14 departamentos de la provincia de Mendoza, con la participación de 38 médicos con formación diabetológica (Grupo de Estudio MENDODIAB-1). Los médicos participantes, junto con los pacientes, previa conformidad oral, completaron una base de datos que cargaron en forma anónima. Cada médico incorporó de manera consecutiva a pacientes con diagnóstico de DM1, según criterio clínico y en tratamiento con insulinización intensiva (basal/bolo o bomba de infusión) iniciada dentro del año de debut. Se los estratificó según IMC en normopeso (IMC 18 a 24,9 kg/m²), sobrepeso (IMC 25 a $29,9 \mathrm{~kg} / \mathrm{m}^{2}$ ) y obesidad 
$\left(\mathrm{IMC} \geq 30 \mathrm{~kg} / \mathrm{m}^{2}\right)$. En los tres grupos se analizaron: edad, circunferencia de cintura entre cresta ilíaca y última costilla, HbA1c, LDL-C <100 mg/dL, HTA (en tratamiento o sin tratamiento con presión arterial sistólica -PAS- $\geq 140 \mathrm{mmHg}$ ó $130 \mathrm{mmHg}$ ) y nivel de instrucción (secundario completo o más).

La actividad física (AF) se definió de acuerdo con su frecuencia en estructurada ( 0 vez/semana, 1 a 2 veces/semana $y \geq 3$ veces/semana), sin incluir tareas del hogar ni tiempo de transporte cotidiano a pie, igual al método usado en el reciente metaanálisis de Bohn et al. ${ }^{13}$.

La sensibilidad a la insulina se evaluó según la TeDG por fórmula de Williams y Orchard: TeDG $(\mathrm{mg} / \mathrm{kg} / \mathrm{min})=21,158+(-0,09 \times \mathrm{CC})+(-3,407 \times \mathrm{HTA})$ $+(-0,551 \times \mathrm{HbA} 1 \mathrm{c})$ (donde $\mathrm{CC}$ es circunferencia de cintura en cm; HTA sí 1, No 0).

\section{Análisis estadístico}

Las variables cualitativas se analizaron por test de chi cuadrado y las cuantitativas por test de ANOVA I corregidas por test de Tukey para comparaciones múltiples. Un valor de $p<0,05$ se consideró estadísticamente significativo. En todos los casos se utilizó un intervalo de confianza del 95\%.

Para tener en cuenta los posibles efectos de confusión sobre la variable de respuesta $\mathrm{HbA} 1 \mathrm{c}$, como edad, duración de la diabetes, edad al inicio de la diabetes, circunferencia de cintura y presión arterial sistólica, se realizó un análisis de regresión logística con el programa MedCalc.

Según la Encuesta Nacional de Factores de Riesgo (ENFR) de 2013, la prevalencia de diabetes en la provincia de Mendoza fue de 8,9\% en mayores de 18 años $^{14}$. Mendoza tiene un total de 1.886 .000 habitantes y el $80 \%$ de la población es mayor de 20 años, lo cual hace una estimación de $\approx 130.000$ adultos con diabetes. Si del 5 al 10\% corresponde a DM1 podría considerarse una cifra de entre $\approx 6.500$ y 13.000 adultos con DM1. Para un universo con ese número de DM1 se definió el tamaño muestral a través del Sample size calculator MedCalc, en 363 a 374 sujetos, con un margen de error del $5 \%$ y un nivel de confianza del $95 \%$.

\section{RESULTADOS}

Se incluyeron $414 \mathrm{DM} 1 \geq 18$ años, mujeres (M) $52 \%$, edad 34,8 $\pm 13,9$ años. Las características de la población según edad, sexo, HbA1c, LDL $\leq 100 \mathrm{mg} / \mathrm{dL}$, HTA, instrucción, TeDG, actividad física $\geq 3 /$ sem según IMC se describen en la Tabla 1.

El sobrepeso presentó el 34,2\% y obesidad el $10 \%$. Las mujeres presentaron mayor frecuencia de normopeso que los varones $(62 \%$ vs $49 \%$; $p=0,01$ ), menor frecuencia de sobrepeso ( $28 \%$ vs $41 \% ; p=0,005)$ y sin diferencia de obesidad $(10 \%$ vs $9,5 \% ; p=0,87$ ) (Figura 1A). Hubo un aumento de sobrepeso/obesidad con la edad $(p<0,0001)$. El incremento fue marcado entre los 25-50 años (Figura $1 \mathrm{~B}$ y C). La PAS $\geq 130 \mathrm{mmHg}$ fue más frecuente en obesidad (54\%) que en sobrepeso (29\%) y que en normopeso (16\%) ( $p<0,0001)$. La TeDG fue de $9,14 \pm 1,64 \mathrm{mg} / \mathrm{kg} / \mathrm{min}$ en los normopeso, $7,62 \pm 1,67 \mathrm{mg} / \mathrm{kg} / \mathrm{min}$ en los sobrepeso y $6,02 \pm 2,20 \mathrm{mg} / \mathrm{kg} / \mathrm{min}$ en los obesos $(p<0,0001)$ (Figura 1D). LDL-C $\leq 100 \mathrm{mg} / \mathrm{dL}$ fue porcentualmente más frecuente en normopeso $(61 \%)$ que en sobrepeso ( $51 \%$ ) y que en obesos $(46 \%)$ sin alcanzar diferencia significativa $(p=0,08)$. No hubo diferencias significativas entre los tres grupos en $\mathrm{HbA} 1 \mathrm{c}$, $A F \geq 3$ veces/semana ni en nivel de instrucción. En el análisis de regresión logística para observar si la antigüedad, la edad, la edad al inicio de la diabetes, la circunferencia de cintura y la presión sistólica representaban un factor de confusión significativo sobre la variable de respuesta $\mathrm{HbA} 1 \mathrm{c}$, ninguna dio riesgo, es decir que la $\mathrm{HbA} 1 \mathrm{c}$ con corte en $\geq 7,9 \%$ fue independiente de dichos factores. 


\begin{tabular}{|c|c|c|c|c|c|c|}
\hline & \multicolumn{2}{|c|}{ Normopeso } & \multicolumn{2}{|c|}{ Sobrepeso } & \multicolumn{2}{|c|}{ Obesidad } \\
\hline Total 414 & \multicolumn{2}{|l|}{$231(55,7 \%)$} & \multicolumn{2}{|l|}{$142(34,2 \%)$} & \multicolumn{2}{|l|}{$41(10 \%)$} \\
\hline Sexo F 215 & $133(61,8 \%)$ & \multirow{2}{*}{$p=0,01$} & $60(27,9 \%)$ & & $22(10,2 \%)$ & \multirow{2}{*}{$\mathrm{p}=0,87$} \\
\hline Sexo M 199 & $98(49,2 \%)$ & & $82(41,2 \%)$ & & $19(9,5 \%)$ & \\
\hline Edad $34,8 \pm 13,9$ & \multicolumn{2}{|l|}{$31,6 \pm 13,2$} & \multicolumn{2}{|l|}{$38,5 \pm 14$} & $40,4 \pm 13,2$ & $p<0,0001$ \\
\hline 18 a 24 N 120 & \multicolumn{2}{|l|}{$76,7 \%$} & \multicolumn{2}{|l|}{$20,0 \%$} & $3,3 \%$ & \multirow{5}{*}{$\mathrm{p}<0,0001$} \\
\hline 25 a 34 N 119 & \multicolumn{2}{|l|}{$52,9 \%$} & \multicolumn{2}{|l|}{$36,1 \%$} & $10,9 \%$ & \\
\hline 35 a 49 N 110 & \multicolumn{2}{|l|}{$46,4 \%$} & \multicolumn{2}{|l|}{$40,9 \%$} & $12,7 \%$ & \\
\hline 50 a $64 \mathrm{~N} 48$ & \multicolumn{2}{|l|}{$33,3 \%$} & \multicolumn{2}{|l|}{$50,0 \%$} & $16,6 \%$ & \\
\hline$+65 N 17$ & \multicolumn{2}{|l|}{$52,9 \%$} & \multicolumn{2}{|l|}{$35,2 \%$} & $11,8 \%$ & \\
\hline $\mathrm{A} 1 \mathrm{c}<7 \%$ & \multicolumn{2}{|l|}{$17,9 \%$} & \multicolumn{2}{|l|}{$25,2 \%$} & $17,0 \%$ & \multirow{3}{*}{$p=0,1039$} \\
\hline$<7,9 \%$ & \multicolumn{2}{|l|}{$29,1 \%$} & \multicolumn{2}{|l|}{$32,3 \%$} & $39,0 \%$ & \\
\hline$\geq 8 \%$ & \multicolumn{2}{|l|}{$52,9 \%$} & \multicolumn{2}{|l|}{$42,4 \%$} & $43,9 \%$ & \\
\hline $\mathrm{AF} \geq 3$ & \multicolumn{2}{|l|}{$35,5 \%$} & \multicolumn{2}{|l|}{$45,7 \%$} & $34,1 \%$ & $p=0,1142$ \\
\hline HTA (>130) 24,4\% & \multicolumn{2}{|l|}{$16,4 \%$} & \multicolumn{2}{|l|}{$28,9 \%$} & $53,6 \%$ & $p<0,0001$ \\
\hline LDL < 100 56,5\% & \multicolumn{2}{|l|}{$61,5 \%$} & \multicolumn{2}{|l|}{$51,5 \%$} & $46,1 \%$ & $\mathrm{p}=0,0809$ \\
\hline Nivel de instrucción $\geq 2^{\circ}$ & \multicolumn{2}{|l|}{$75,7 \%$} & \multicolumn{2}{|l|}{$81,6 \%$} & $80,48 \%$ & $p=0,3768$ \\
\hline $\begin{array}{l}\text { Tasa estimada de } \\
\text { disposición de glucosa }\end{array}$ & \multicolumn{2}{|c|}{$9,14 \pm 1,64 \mathrm{mg} / \mathrm{kg} / \mathrm{min}$} & $7,62 \pm 1,67 \mathrm{~m}$ & $\min$ & $6,02 \pm 2,20 \mathrm{mg} / \mathrm{kg} / \mathrm{min}$ & $p<0,0001$ \\
\hline
\end{tabular}

Tabla 1: Edad, sexo, A1c, LDL $\leq 100$ mg/dL, HTA, instrucción, TeDG, actividad física $\geq 3 /$ semana según IMC.

\begin{tabular}{|l|l|l|l|l|}
\hline \multirow{2}{*}{} & \multicolumn{2}{|c|}{ Población general (ENFR 2013) } & \multicolumn{2}{c|}{ DM1 Mendoza N=414 } \\
\cline { 2 - 5 } & Sobrepeso\% & Obesidad\% & Sobrepeso\% & Obesidad\% \\
\hline Total & 37,1 & 20,8 & 34,2 & 10,0 \\
\hline Varones & 43,3 & 22,9 & 41,2 & 9,5 \\
\hline Mujeres & 31,3 & 18,8 & 27,9 & 10,2 \\
\hline 18 a 24 años & 25,4 & 7,7 & 20,0 & 3,3 \\
\hline 25 a 34 años & 35,0 & 15,8 & 36,1 & 10,9 \\
\hline 35 a 49 años & 39,8 & 24,3 & 40,9 & 12,7 \\
\hline 50 a 64 años & 40,9 & 29,6 & 50,0 & 16,6 \\
\hline$\geq 65$ años & 42,6 & 24,3 & 35,2 & 11,8 \\
\hline
\end{tabular}

Tabla 2: Descripción de normopeso, sobrepeso y obesidad en DM1 y población general según ENFR 2013. 


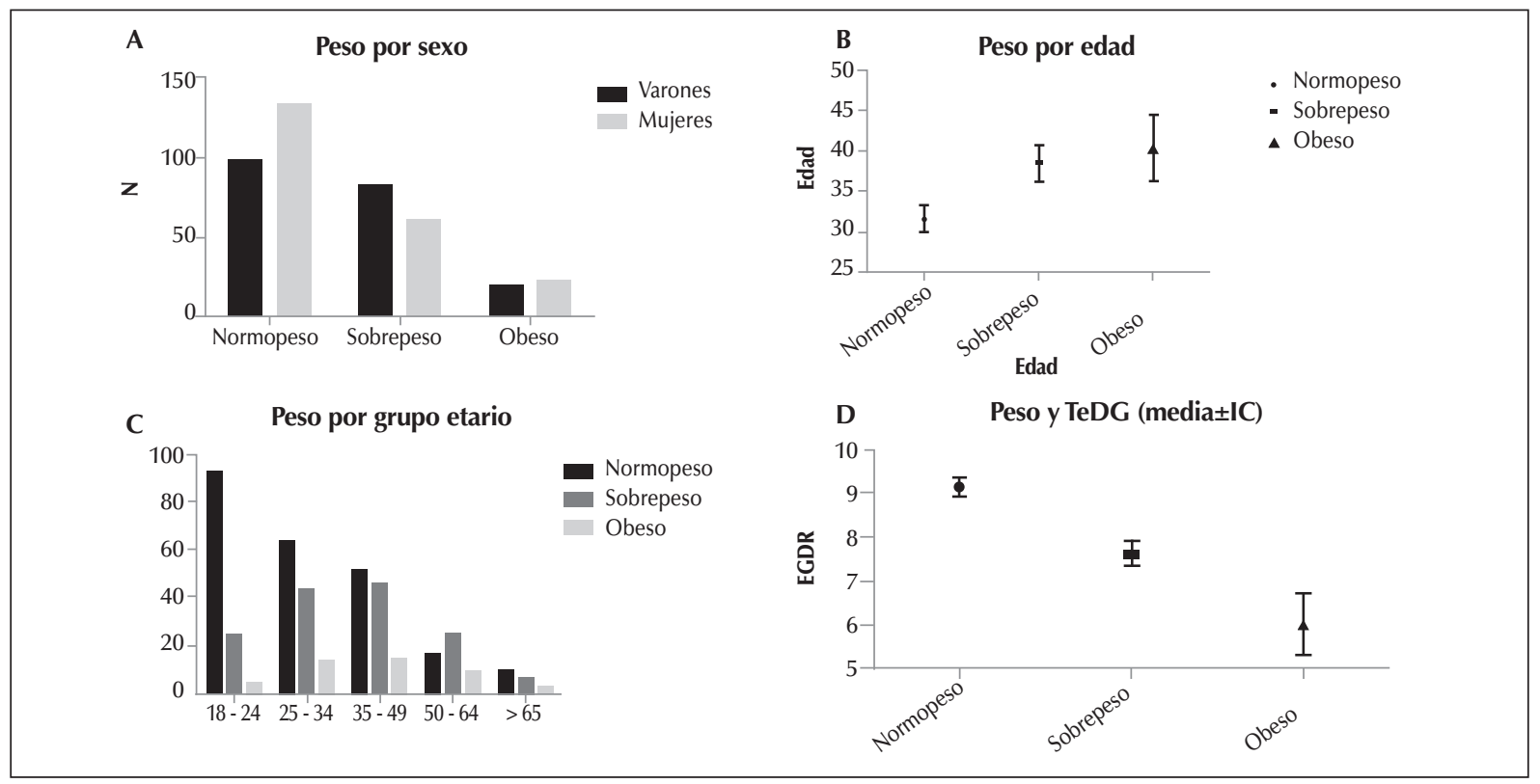

N: número absoluto de pacientes.

Figura 1 (A, B, C, D): Peso por: A sexo, B edad, C grupo etario, D TeDG.

\section{DISCUSIÓN Y CONCLUSIONES}

Se conoce que la obesidad contribuye a la fisiopatología y a las complicaciones de la DM2. Múltiples guías enfatizan el manejo de la obesidad tanto en individuos DM2 como en prediabéticos, dada la fuerte evidencia que demuestra que el tratamiento de la obesidad puede demorar el comienzo de la DM2 y mejorar el control glucémico. La obesidad, antes infrecuente en la DM1, es ahora un problema creciente. La situación actual muestra que no es lo suficientemente jerarquizada, además de pobremente entendida y abordada ${ }^{19}$.

Encontramos sobrepeso en el 34,2\% y obesidad en el 10\%. En Mendoza por la ENFR 2013, en la población general, la prevalencia de obesidad en mayores de 18 años fue del 20,5\%, similar al $20,8 \%$ a nivel nacional. En nuestra población DM1 se advirtió similitud en la frecuencia de sobrepeso y un $50 \%$ menor de obesidad, en general y por grupos etarios (Tabla 2). Al igual que en la población general, el sobrepeso fue mayor en los varones y la obesidad similar en ambos sexos.

La prevalencia de HTA en nuestra población DM1 fue de $24,4 \%$ (PAS $\geq 130 \mathrm{mmHg}$ ) y $21,4 \%$ (PAS $\geq 140 \mathrm{mmHg}$ ). El Estudio Europeo de Pacientes con Diabetes Mellitus Insulinodependiente (EURODIAB IDDM) sobre 3.250 DM1 de 16 países europeos, con una edad media de $32,7 \pm 10$ años, mostró una prevalencia de HTA (PAS $\geq 140 \mathrm{mmHg}$ ) de $24,0 \%{ }^{15}$. Gagliardino y col., en la séptima ola del International Diabetes Management Practices Study (IDMPS), encontraron en 2.000 DM1 de países de cinco continentes, con 34 años de edad media, una prevalencia de 19,9\% de pacientes tratados o con PA $\geq 130 / 80 \mathrm{mmHg}^{16}$. En nuestra población la HTA se asoció fuertemente con sobrepeso y obesidad $(28,9$ y $53,6 \%$ respectivamente) en comparación con el $16,4 \%$ en los sujetos con normopeso.

En el mismo estudio de Gagliardino y col. el 50,7\% de los sujetos DM1 tenía un LDL-C $\leq 100 \mathrm{mg} / \mathrm{dL}$. En nuestra población DM1 el 56,5\% presentó $\mathrm{LDL}-\mathrm{C} \leq 100 \mathrm{mg} / \mathrm{dL}$. En los sujetos con normopeso el LDL-C $\leq 100 \mathrm{mg} / \mathrm{dL}$ fue de $61,5 \%$, en los sujetos con sobrepeso de $51,5 \%$ y en obesos de $46,1 \%$. Si bien la diferencia no alcanzó significación estadística $(p=0,08)$, podría considerarse una tendencia clínicamente significativa.

La tasa estimada de disposición de glucosa (TeDG) es un método basado en parámetros clínicos que incluyen presencia de HTA, hemoglobina glicosilada $(\mathrm{HbA} 1 \mathrm{c})$ y la relación cintura-cadera, y ha sido validada con clamp euglucémico hiperinsulinémico por Williams y Orchard et al. en pacientes con DM1 para determinar el grado de sensibilidad a la insulina ${ }^{17}$. Cuando usaron circunferencia de cintura en lugar de la relación cintura-cadera, se mantuvo un patrón similar. En este estudio hubo una clara correlación negativa entre el peso y la TeDG, siendo de 9,14 $\pm 1,64 \mathrm{mg} / \mathrm{kg} / \mathrm{min}$ en DM1 
con normopeso, 7,62 $\pm 1,67 \mathrm{mg} / \mathrm{kg} / \mathrm{min}$ en sujetos con sobrepeso y $6,02 \pm 2,20 \mathrm{mg} / \mathrm{kg} / \mathrm{min}$ en obesos.

La obesidad, HTA e insulinorresistencia integran el síndrome metabólico junto con la dislipidemia y disglucemia. Las complicaciones como la enfermedad cardiovascular y la nefropatía son más frecuentes en DM1 con síndrome metabólico asociado ${ }^{18}$. Más aún los pacientes DM1 con retinopatía y neuropatía tienen baja TeDG lo cual sugiere una relación entre sensibilidad a la insulina y complicaciones microvasculares ${ }^{19}$.

La acumulación de datos de prevalencia y las consecuencias del sobrepeso/obesidad en pacientes con DM1 son evidentes, sin embargo esta comorbilidad es subvalorada. El sobrepeso/obesidad es probable debido a los cambios en el medio ambiente que afectan a la población en general y una consecuencia no deseada de los enfoques modernos y más intensivos para el manejo de la hiperglucemia en DM1, como ya lo demostraron el Diabetes Control and Complications Trial (DCCT) y otros estudios. No se realizaron ensayos clínicos controlados aleatorios que evalúen los enfoques dietéticos para optimizar a la vez tanto el control del peso como el control glucémico en DM120.

En el reconocido estudio y posterior seguimiento Diabetes Control and Complications Trial/ Epidemiology of Diabetes Interventions and Complications (DCCT/EDIC), a los 14 años, los eventos cardiovasculares en el grupo de tratamiento intensivo con mayor ganancia de peso fueron significativamente más frecuentes que en el grupo intensivo sin cambios o con mínima ganancia de peso, e indistinguibles de la tasa de eventos en el grupo control no intensivo ${ }^{21}$.

Sin dudas los pacientes que cumplen con las pautas de actividad física tienen una mejor composición corporal, niveles más bajos de HbA1c y un mejor perfil lipídico. Por lo tanto, el aumento de los niveles de actividad física podría mejorar drásticamente la salud de las personas con DM1 y sobrepeso/obesidad. Existen recientes publicaciones que proporcionan los parámetros de ejercicio más actualizados para personas con DM1 con respecto a objetivos glucémicos, seguridad, eficacia, nutrición e insulina para evitar complicaciones, así como las barreras para el ejercicio ${ }^{22,23}$. Nuestra población no mostró diferencias en la frecuencia de actividad física entre obesos y normopeso siendo baja en ambos grupos; sólo el 35,5\% de los normopeso y el $34,1 \%$ de los obesos refirieron hacer actividad fí- sica tres o más veces por semana. El ejercicio para descender de peso requiere estrategias que hagan foco en la reducción de las concentraciones sanguíneas de insulina durante y luego del ejercicio, así como evitar el consumo de colaciones.

Además de los conocidos efectos cardiometabólicos, también el sobrepeso/obesidad, especialmente en jóvenes DM1, pueden generar el riesgo de conductas mal adaptativas. Se estima que del 20 al 40\% de los jóvenes con DM1 se han involucrado en la manipulación de la insulina (omisión de dosis) para prevenir el aumento de peso ${ }^{24}$, y se ha demostrado que la manipulación de la insulina persiste hasta la edad adulta. El sexo femenino, el IMC más alto, las comidas familiares poco frecuentes, la alta atención familiar al peso, la depresión, la baja autoestima y la insatisfacción corporal se asocian con un mayor riesgo de conductas en las cuales la restricción de insulina se usa como un medio para controlar el peso corporal en personas con $\mathrm{DM} 1^{25}$.

En otro orden, dado que la obesidad se vincula estrechamente con la enfermedad grasa hepática no alcohólica (EGHNA) es razonable postular que la obesidad en DM1 tendrá un impacto negativo en la salud hepática. Algunos estudios muestran que cuando la EGHNA se presenta en forma concurrente con DM1 existe un incremento de complicaciones cardiovasculares ${ }^{26}$, renale ${ }^{27}$ y neuropáticas ${ }^{28}$.

Las terapias farmacológicas adjuntas a la insulina se plantean para mejorar el control glucémico y agregar potenciales beneficios en obesidad y factores de riesgo cardiovascular. Metformina, en el estudio Reducing with Metformin Vascular Adverse Lesions (REMOVAL), generó pequeñas reducciones en el peso y en lípidos, pero no mejoró $\mathrm{A} 1 \mathrm{c}^{29}$. Un reciente metaanálisis de siete estudios controlados, la mayoría pequeños y de corta duración, mostró que el agregado a insulina de GLP1-AR disminuyó modestamente A1c $(-0,21 \%)$ y el peso corporal $(-3,5 \mathrm{~kg})^{30}$. Limitados datos existen con los SGLT2i, pero los reportes, como el estudio Dapagliflozin Evaluation in Patients With Inadequately Controlled Type 1 Diabetes (DEPICT-1) ${ }^{31}$ sugieren mejoría en A1c, peso y circunferencia de cintura. Queda por definir el riesgo de cetoacidosis diabética "euglucémica" asociada a estos fármacos. Con DPP4i hay datos limitados sin beneficios en A1c, pero con algo de reducción de dosis de insulina ${ }^{32}$. Se necesitan futuras investigaciones para determinar la eficacia y seguridad de estos fármacos. 
Nuestra muestra de 414 DM1 se compuso de pacientes que estaban en seguimiento por médicos con experiencia en la atención de personas con DM1. Por ello no podemos generalizar nuestros resultados al total de DM1 de Mendoza; sin embargo, el sistema provincial estatal de salud provee asistencia a las personas con DM1 por profesionales capacitados. El $77 \%$ de la muestra correspondió a pacientes con cobertura médica por obra social o prepago, y el $23 \%$ con cobertura por parte de la Seguridad Pública. En Argentina se estima que el $36 \%$ de la población corresponde a la Seguridad Pública ${ }^{33}$.

En conclusión, en este estudio la frecuencia de sobrepeso/obesidad en DM1 adultos (44,2\%) fue alta y similar a observaciones recientes. El incremento fue marcado entre los 25-50 años y se asoció fuertemente con HTA e insulinorresistencia. Existe una urgente necesidad por desarrollar guías específicas para la prevención y el tratamiento de la obesidad con buen control glucémico en pacientes con DM1. El desarrollo de estas estrategias requerirá de un enfoque transdisciplinario que incluya a epidemiólogos, diabetólogos, nutriólogos, fisiólogos del ejercicio, psicólogos y educadores.

\section{Miembros del Grupo de Estudio de Diabetes Tipo 1 de Mendoza (MENDODIAB)}

Martín Rodríguez, Edgardo Trinajstic, Alejandra Cicchitti, Joaquín González, Celina Bertona, Víctor Previtera, Nelson Rodríguez, Pedro Calella, Pablo Ávila, Soledad Acosta, María Inés Argerich, Carolina Dromi, Patricia Lemos, Franco Bellomo, Laura Bidot, Karina Mengoni, Daniel Giorgini, Liliana Abdala, Gabriela Negri, Carla Ponce, Luciano Ortiz, Eligio Negri, Macarena Argumedo, Gabriel Minuchín, Laura Dimov, Luis Lombardo, Paula Muñoz, Romina Sosa, Zelmira Guntsche, Roxana Abeledo, Laura Cuello, Alfredo Bonadé, Norma Carrasco, Leticia Barrera, Raúl David, Laura Romero, Luis Biliato, Hugo Lavandaio.

\section{BIBLIOGRAFÍA}

1. Kjaer IGH, Kolle E, Hansen BH, Anderssen SA, Torstveit MK. Obesity prevalence in Norwegian adults assessed by body mass index, waist circumference and fat mass percentage. Clinical Obesity 2015; 5:211-8.

2. Szadkowska A, Madej A, Ziolkowska K, Szymanska M, Jeziorny K, Mianowska B, et al. Gender and age-dependent effect of type 1 diabetes on obesity and altered body composition in young adults. Annals of Agricultural and Environmental Medicine $2015 ; 22: 124-8$.
3. Libman IM, Becker DJ. Coexistence of type 1 and type 2 diabetes mellitus: "double" diabetes? Pediatric Diabetes 2003; 4:110-3.

4. Pozzilli P, Guglielmi C, Caprio S, Buzzetti R. Obesity, autoimmunity, and double diabetes in youth. Diabetes Care 2011 May; 34 Suppl: S166-70.

5. Petrie D, Lung TW, Rawshani A, Palmer AJ, Svensson AM, Eliasson B, Clarke P. Recent trends in life expectancy for people with type 1 diabetes in Sweden. Diabetologia 2016; 59:1167-76.

6. Rawshani A, Rawshani A, Franzén S, Eliasson B, Svensson AM, Miftaraj M, McGuire DK, Sattar N, Rosengren A, Gudbjörnsdottir S. Mortality and cardiovascular disease in type 1 and type 2 diabetes. N Engl J Med 2017; 376:1407-18.

7. Minges KE, Whittemore R, Grey M. Overweight and obesity in youth with type 1 diabetes. Annu Rev Nurs Res 2013; 31:47-69.

8. Liu LL, Lawrence JM, Davis C, Liese AD, Pettitt DJ, Pihoker C Dabelea D, Hamman R, Waitzfelder B, Kahn HS; SEARCH for Diabetes in Youth Study Group. Prevalence of overweight and obesity in youth with diabetes in USA: the SEARCH for Diabetes in Youth study. Pediatr Diabetes 2010; 11: 4-11.

9. Conway B, Miller RG, Costacou T, Fried L, Kelsey S, Evans RW, Orchard TJ. Temporal patterns in overweight and obesity in type 1 diabetes. Diabet Med 2010; 27:398-404.

10. Price SA, Gorelik A, Fourlanos S, Colmana PG, Wentworth JM. Obesity is associated with retinopathy and macrovascular disease in type 1 diabetes. Obesity Research \& Clinical Practice 2014; 8:e178-e182.

11. Vestberg D, Rosengren A, Olsson M, Gudbjörnsdottir S, Svensson AM, Lind M. Relationship between overweight and obesity with hospitalization for heart failure in 20.985 patients with type 1 diabetes. Diabetes Care 2013; 36: 2857-2861.

12. Caeiro G, Romero-González SA, Itria J, Waitman J. Type 1 diabetes and insulin resistance: estimated glucose disposal rate and its correlation with chronic complications. Revista de la Sociedad Argentina de Diabetes 2017; 51: 38-46.

13. Bohn B, Herbst A, Pfeifer M, Krakow D, Zimny S, Kopp F, et al.; for the DPV Initiative. Impact of physical activity on glycemic control and prevalence of cardiovascular risk factors in adults with type 1 diabetes: a cross-sectional multicenter study of 18.028 patients. Diabetes Care 2015; 38:1536-1543.

14. Tercera Encuesta Nacional de Factores de Riesgo para Enfermedades NoTransmisibles. 10 de Julio de 2015. Buenos Aires, Argentina. ISBN: 978-950-38-0218-2.

15. Collado-Mesa F, Colhount HM, Stevenst LK, Boavida J, Fullert $\mathrm{J}$; the EURODIAB IDDM Complications Study. Prevalence and management of hypertension in type 1 diabetes mellitus in Europe: the EURODIAB IDDM Complications Study. Diabet Med 1999; 16: 41-49.

16. Gagliardino JJ, Aschner $P$, Likova $H$, Lavalle F, Ramachandran A, Kaddaha G, Mbanya JC, Shestakova M, Chantelot JM, Chan J. Frequency of high blood pressure and dyslipidemia in T1D and T2D: results from the International Diabetes Management Practices Study (IDMPS). ADA 2018: 621-P.

17. Williams KV, Erbey JR, Becker D, Arslanian S, Orchard TJ 2000 Can clinical factors estimate insulin resistance in type 1 diabetes? Diabetes 2000; 49: 626-632.

18. Pambianco G, Costacou T, Orchard TJ. The prediction of major outcomes of type 1 diabetes: a 12 year prospective evaluation of three separate definitions of the metabolic syndrome and their components and estimated glucose disposal rate: the Pittsburgh Epidemiology of Diabetes Complications Study experience. Diabetes Care 2007; 30:1248-1254. 
19. Chillarón JJ, Goday A, Flores-Le-Roux JA, Benaiges D, Carrera MJ, Puig J, Cano-Pérez JF, Pedro-Botet J. Estimated glucose disposal rate in assessment of the metabolic syndrome and microvascular complications in patients with type 1 diabetes. $\mathrm{J}$ Clin Endocrinol Metab 2009; 94:3530-3534.

20. Corbin KD, Driscoll KA, Pratley RE, Smith SR, Maahs DM, Mayer-Davis EJ; on behalf of Advancing Care for Type 1 Diabetes and Obesity Network (ACT1ON). Obesity in type 1 diabetes: pathophysiology, clinical impact, and mechanisms. Endocrine Reviews 2018; 39:629-663.

21. Purnell JQ, Braffett BH, Zinman B, Gubitosi-Klug R A, Sivitz W, Bantle JP, Ziegler G, Cleary PA, Brunzell JD. Impact of excessive weight gain on cardiovascular outcomes in type 1 diabetes: results from the Diabetes Control and Complications Trial/ Epidemiology of Diabetes Interventions and Complications (DCCT/EDIC) study. Diabetes Care 2017; 40:1756-1762.

22. Riddell MC, Gallen IW, Smart CE, Taplin CE, Adolfsson P, Lumb AN, Kowalski A, Rabasa-Lhoret R, McCrimmon RJ, Hume C, Annan F, Fournier PA, Graham C, Bode B, Galassetti P, Jones TW, Millan IS, Heise T, Peters AL, Petz A, Laffel LM. Exercise management in type 1 diabetes: a consensus statement. Lancet Diabetes Endocrinol 2017; 5:377-390.

23. Rodríguez M. Nutrición y ejercicio en las personas con diabetes. Rev ALAD 2017; 7: 40-49

24. Hanlan ME, Grffith J, Patel N, Jaser SS. Eating disorders and disordered eating in type 1 diabetes: prevalence, screening, and treatment options. Curr Diab Rep 2013; 13:909-916.

25. Goebel-Fabbri AE, Fikkan J, Franko DL, Pearson K, Anderson $\mathrm{BJ}$, Weinger $\mathrm{K}$. Insulin restriction and associated morbidity and mortality in women with type 1 diabetes. Diabetes Care 2008; 31:415-419.

26. Targher G, Bertolini L, Padovani R, Rodella S, Zoppini G, Pichiri I, Sorgato C, Zenari L, Bonora E. Prevalence of non-alcoholic fatty liver disease and its association with cardiovascular disease in patients with type 1 diabetes. J Hepatol 2010; 53: 713-718.
27. Targher G, Mantovani A, Pichiri I, Mingolla L, Cavalieri V, Mantovani W, Pancheri S, Trombetta M, Zoppini G, Chonchol M, Byrne CD, Bonora E. Nonalcoholic fatty liver disease is independently associated with an increased incidence of chronic kidney disease in patients with type 1 diabetes. Diabetes Care 2014; 37:1729-1736.

28. Mantovani A, Rigolon R, Mingolla L, Pichiri I, Cavalieri V, Salvotelli L, Stoico V, Zoppini G, Bonora E, Targher G. Nonalcoholic fatty liver disease is associated with an increased prevalence of distal symmetric polyneuropathy in adult patients with type 1 diabetes. J Diabetes Complications 2017; 31:1021-1026.

29. Petrie JR, Chaturvedi N, Ford I, et al.; REMOVAL Study Group. Cardiovascular and metabolic effects of metformin in patients with type 1 diabetes (REMOVAL): a double-blind, randomised, placebo-controlled trial. Lancet Diabetes Endocrinol 2017; 5:597-609

30. Wang W, Liu H, Xiao S, Liu S, Li X, Yu P. Effects of insulin plus glucagon-like peptide-1 receptor agonists (GLP-1RAs) in treating type 1 diabetes mellitus: a systematic review and metaanalysis. Diabetes Ther 2017; 8:727-738.

31. Dandona P, Mathieu C, Phillip M, Hansen L, Griffen SC, Tschöpe D, Thorén F, Xu J, Langkilde AM; on behalf of the DEPICT-1 Investigators. Efficacy and safety of dapagliflozin in patients with inadequately controlled type 1 diabetes (DEPICT-1): 24-week results from a multicentre, double-blind, phase 3 , randomised controlled trial. Lancet Diabetes Endocrinol 2017; 5: 864-76.

32. Guo H, Fang C, Huang Y, Pei Y, Chen L, Hu J. The efficacy and safety of DPP4 inhibitors in patients with type 1 diabetes: a systematic review and metanalysis. Diabetes Res Clin Pract 2016; 121:184-191.

33. Organización Mundial De La Salud. Informe Argentina 2017. Disponible en: http://administracionsalud.com.ar/organizacion-mundial-de-la-salud-informe-argentina-2017. 